\title{
A NOTE ON SELECTING THE BETTER BINOMIAL POPULATION
}

\author{
S.S. CHITGOPEKAR \\ DEPARTMENT OF MANAGEMENT \& MARKETING \\ ILLINOIS STATE UNIVERSITY \\ NORMAL, ILLINOIS 61761
}

(Received August 31, 1981 and in revised form August 23, 1982)

\begin{abstract}
An inverse sampling procedure is proposed for the problem of selecting the better of two treatments when the responses are dichotomous. This procedure is particularly useful when it is desired to limit the number of failures during the decision making stage. The regret function of the procedure is derived and it is shown that this procedure has a minimax regret property when compared to a fixed sample procedure studied by Pradhan and Sathe [2]. Numerical evidence indicates that this procedure dominates the fixed sample procedure of Pradhan and Sathe over the entire parameter space.
\end{abstract}

SUBJECT AREAS: Statistics, Inverse Sampling.

1980 MATHEMATICS SUBJECT CLASSIFICATION CODES: 62F07, $62 \mathrm{~L} 99$.

\section{INTRODUCTION.}

Canner [1] and Pradhan and Sathe [2] have studied the problem of selecting the better of two treatments, $\mathrm{T}_{1}$ and $\mathrm{T}_{2}$, when a total of $\mathrm{N}$ (present or future) patients are to be treated for a certain disease with one of the two treatments. Both articles study the following decision procedure (CPS - procedure) which involves a fixed sample: each treatment is given to $n(n \leq N / 2)$ patients; the response to a treatment is observed as success or failure. After $n$ observations with each treat- 
ment, the one with more successes is chosen as the better treatment; (in case of a tie, one of the two treatments is selected as the better one at random with equal probability) and then that treatment is given to the remaining ( $\mathrm{N}-2 \mathrm{n})$ patients. In this note we study the same problem but with an inverse sampling procedure. We assume that $\mathrm{N}$ is an even integer, say $\mathrm{N}=2 \mathrm{~m}$, and the $\mathrm{N}$ patients are divided into m pairs. The two treatments are applied to each pair (assignment of the two patients in any pair to the treatments being random) successively until $k$ failures are observed with any one treatment. Then the other treatment is selected as the better treatment. If exactly $k$ failures are observed on both the treatments simultaneously or if less than $\mathrm{k}$ failures are observed on both the treatments until all the $\mathrm{N}$ patients are treated, then one of the two treatments is selected as the better treatment at random with equal probability. The treatment selected as the better one is then applied to the remaining (if any) patients.

Several authors, e.g., Sobel and Weiss [3], have considered stopping rules based on the difference in the number of successes (and/or failures) with the two treatments. For $\theta_{1}$ and/or $\theta_{2}$ small, (where $\theta_{i}$ denotes the probability of success with the $1^{\text {th }}$ treatment) such procedures result in larger expected number of patients treated and/or larger expected number of patients receiving the poorer treatment during the decision making stage. Our approach ensures that a decision is reached with at most $2 k$ failures. In situations where an early (but correct) decision is desired with minimal number of failures during the decision making stage, this approach would be better as it enables us to control that number of failures. Our approach can be thought of as the "truncated" version of the situation where the two treatments can be applied to an infinite number of patients.

In this article we study the particular case of $k=1$. The more general case will be treated in a separate article.

\section{PROPERTIES OF THE PROCEDURE.}

\subsection{Probability of Correct Selection}

Let $\theta_{i}$ denote the probability of success and $N_{i}$ the number of trials to the first failure with treatment $T_{i}(i=1,2)$. Without loss of generality, throughout 
this article, we assume that $\theta_{1}>\theta_{2}$. Let the probability of correctly selecting $T_{1}$ as the better treatment be denoted by $P=P\left(\theta_{1}, \theta_{2}, m\right)$. Then we have

$$
\begin{aligned}
P & =1 / 2\left[\sum_{r=1}^{m} \operatorname{Pr}\left(N_{1}=N_{2}=r\right)+\operatorname{Pr}\left(N_{1}>m, N_{2}>m\right)\right]+\operatorname{Pr}\left(N_{2}<N_{1} \leq m\right) \\
& =1 / 2\left[\sum_{r=1}^{m} \Theta_{1}^{r-1} \Theta_{2}^{r-1}\left(1-\Theta_{1}\right)\left(1-\Theta_{2}\right)+\dot{\theta}_{1}{ }^{m} \Theta_{2}{ }^{m}{ }^{m}+\sum_{r=1}^{m} \Theta_{1}^{r} \Theta_{2}^{r-1}\left(1-\Theta_{2}\right)\right. \\
& =1 / 2\left[1+\left(\Theta_{1}-\Theta_{2}\right)\left\{1-\left(\Theta_{1} \Theta_{2}\right)^{m}\right\} /\left(1-\Theta_{1} \Theta_{2}\right)\right] .
\end{aligned}
$$

Observe that $P \geq 1 / 2$ as it should be. If $P^{*}=P^{*}\left(\theta_{1}, \theta_{2}\right)=$ $\mathrm{m} \stackrel{1}{\rightarrow}$ im $\mathrm{P}\left(\Theta_{1}, \Theta_{2}, \mathrm{~m}\right)$ then we have

$$
\mathrm{P}^{*}=1 / 2\left[1+\left(\Theta_{1}-\Theta_{2}\right) /\left(1-\Theta_{1} \Theta_{2}\right)\right] \text {. }
$$

Also notice that for fixed $\left(\Theta_{1}-\Theta_{2}\right)$, P is an increasing function of $\left(\Theta_{1} \Theta_{2}\right)$.

\section{2 Expected Sample Size}

Let $\mathrm{S}$ denote the number of patients treated before a decision as to the better treatment is reached. Then

$$
\begin{aligned}
S & =2 r<2 m & & \text { if } N_{i} \geq N_{j}=r(i, j=1,2 ; i \neq j) . \\
& =2 m & & \text { if } N_{1} \geq m \text { and } N_{2} \geq m .
\end{aligned}
$$

Using the tail probability representation of expectation we have

$$
\begin{aligned}
\mathrm{E}(\mathrm{S})= & \underset{\mathrm{r}=1}{\mathrm{~m}} \operatorname{Pr}(\mathrm{S} \geq 2 \mathrm{r}) \\
= & \underset{\mathrm{r}=1}{\mathrm{~m}} \operatorname{Pr}\left(\mathrm{N}_{1} \geq \mathrm{r}, \mathrm{N}_{2} \geq \mathrm{r}\right) \\
= & \underset{\mathrm{r}=1}{\mathrm{~m}} \Theta_{1}{ }^{\mathrm{r}} \Theta_{2} \mathrm{r}=2\left\{1-\left(\Theta_{1} \Theta_{2}\right)^{\mathrm{m}}\right\} /\left(1-\Theta_{1} \Theta_{2}\right)
\end{aligned}
$$

Observe that $\mathrm{E}(\mathrm{S})$ depends on $\Theta_{1}$ and $\Theta_{2}$ only through $\left(\Theta_{1} \Theta_{2}\right)$ and is increasing in $\left(\Theta_{1} \Theta_{2}\right)$

\subsection{Regret Function}

We define the regret function, $R\left(\theta_{1}, \Theta_{2}, m\right)$, as the expected number of failures in the $2 \mathrm{~m}$ patients with our proposed decision procedure in excess of the expected number of failures if all the $2 \mathrm{~m}$ patients had received the better treatment.

The expected number of failures when all the $2 \mathrm{~m}$ patients receive the better 
treatment is $2 m\left(1-\theta_{1}\right)$. It can be easily verified that the expected number of failures in the $2 \mathrm{~m}$ patients with our decision procedure is

$$
\frac{\left(\Theta_{1}-\Theta_{2}\right)^{2}\left\{1-\left(\Theta_{1} \Theta_{2}\right)^{m}\right\}}{\left(1-\Theta_{1} \Theta_{2}\right)^{2}}+\frac{m\left(1-\Theta_{1}\right)\left(1-\Theta_{2}\right)\left(2+\Theta_{1}+\Theta_{2}\right)}{\left(1-\Theta_{1} \Theta_{2}\right)}
$$

Hence

$$
R\left(\theta_{1}, \theta_{2}, \mathrm{~m}\right)=\frac{\left(\theta_{1}-\theta_{2}\right)^{2}\left\{1-\left(\theta_{1} \theta_{2}\right)^{\mathrm{m}}\right\}}{\left(1-\theta_{1} \theta_{2}\right)^{2}}+\mathrm{m}\left(\theta_{1}-\theta_{2}\right)\left[1-\frac{\Theta_{1}-\theta_{2}}{1-\theta_{1} \theta_{2}}\right]
$$

After some algebraic simplification (2.4) can be written in the following form:

$$
R\left(\theta_{1}, \theta_{2}, m\right)=\left(\theta_{1}-\theta_{2}\right)\left[1 / 2 E(S)+\{2 m-E(S)\}\left(1-P^{*}\right)\right] .
$$

\section{COMPARISON WITH CPS - PROCEDURE}

We shall compare our procedure with the CPS - procedure in terms of the respective regret functions. The regret function obtained in Pradhan and Sathe [2] 1s:

$$
R^{-}\left(\theta_{1}, \theta_{2}, m\right)=\left(\theta_{1}-\theta_{2}\right)[n+(N-2 n) U]
$$

where $U$ is their probability of wrong selection (given by their equation (2.1)) and $2 n$ ( $f$ ixed) is the number of patients treated before a decision is reached. We thus see that (2.5) and (3.1) are similar in structure which facilitates their comparison.

Observe that for our procedure, the sample size, $S$, before a decision is reached is a random variable whereas in Pradhan and Sathe [2], the sample size $2 \mathrm{n}$ is fixed. To make the comparison a just one, we set $2 n=E(S)$. In this situation, we see that $R\left(\theta_{1}, \theta_{2}, m\right) \leq R^{-}\left(\theta_{1}, \theta_{2}, m\right)$ whenever $P^{*} \geq 1-U$.

In general it is not analytically easy to find the region in the $\left(\theta_{1}, \theta_{2}\right)-$ space where $P^{*} \geq 1-U$ inequality is satisfied. However, we can say something about the inequality as $\theta_{1} \rightarrow 1$.

THEOREM 3.1 In the neighborhood of $\theta_{1}=1, R\left(\theta_{1}, \theta_{2}, m\right) \leq R^{-}\left(\theta_{1}, \theta_{2}, m\right)$ if $2 n$ $-E(S)$.

PROOF: For $\theta_{1}=1$ and $0<\theta_{2}<1$ we have $P^{*}=1$ while $U=1 / 2 \theta_{2}^{\mathrm{n}}>0$. Hence $P^{*}>1-U$. Since $R\left(\theta_{1}, \theta_{2}, m\right)$ and $R^{-}\left(\theta_{1}, \theta_{2}, m\right)$ are continuous functions of $\left(\theta_{1}, \theta_{2}\right)$, the theorem follows. 
In the next theorem we show that our procedure has minimax regret when compared to the CPS - procedure.

THEOREM 3.2: For $2 \mathrm{n}=\mathrm{E}(\mathrm{S})$ and $\theta_{1}-\theta_{2}=\delta>0$ where $\delta$ is arbitrary but fixed, we have

$$
\begin{aligned}
& \max R\left(\theta_{1}, \theta_{2}, \mathrm{~m}\right) \leq \max R^{-}\left(\theta_{1}, \theta_{2}, \mathrm{~m}\right) \\
& \theta_{1}, \theta_{2} \quad \theta_{1}, \theta_{2}
\end{aligned}
$$

for all $\mathrm{m} \geq 1$

To prove this theorem we first need the following Lemma:

LEMMA 3.1: For fixed $\left(\Theta_{1}-\theta_{2}\right)$ and $m, R\left(\theta_{1}, \theta_{2}, m\right)$ is a decreasing function of $\left(\Theta_{1} \Theta_{2}\right)$

PROOF: Letting $\theta_{1} \theta_{2}=x,(2.4)$ can be rewritten as

$$
R\left(\Theta_{1}, \quad \theta_{2}, m\right)=m\left(\theta_{1}-\theta_{2}\right)+\left(\theta_{1}-\theta_{2}\right)^{2}\left\{\left(1-x^{m}\right) /(1-x)^{2}-m /(1-x)\right\} .
$$

To prove the lemma it suffices to show that $g(x)=\left(1-x^{m}\right) /(1-x)^{2}-m /(1-x)$ is decreasing in $x$ which can be seen to be the case by writing

$$
\begin{aligned}
g(x) & =\left(1+x+\ldots+x^{m-1}-m\right) /(1-x) \\
& =-\sum_{i=1}^{m-1}\left(\frac{1-x^{i}}{1-x}\right) \\
& =-\sum_{i=1}^{m-1}\left(1+x+\ldots \cdot x^{i-1}\right) .
\end{aligned}
$$

This proves the lemma.

To prove Theorem $3.2 \mathrm{fix} \Theta_{1}-\Theta_{2}=\delta>0$. Then by Lemma 3.1 we have

$$
\max _{\Theta_{1}, \Theta_{2}} R\left(\Theta_{1}, \Theta_{2}, \mathrm{~m}\right)=\mathrm{R}(\delta, 0, \mathrm{~m})=\delta^{2}+\mathrm{m} \delta(1-\delta)
$$

For $\Theta_{1}=\delta, \Theta_{2}=0$ we have $E(S)=2$. For $n=1 / 2 E(S)=1$ we $f$ ind that

$$
\begin{gathered}
\mathrm{R}^{\prime}(\delta, \circ, \mathrm{m})=\delta^{2}+\mathrm{m} \delta(1-\delta)=\max \mathrm{R}\left(\Theta_{1}, \Theta_{2}, \mathrm{~m}\right) . \\
\Theta_{1}, \Theta_{2} \\
\max \mathrm{R}^{\prime}\left(\Theta_{1}, \Theta_{2}, \mathrm{~m}\right) \geq \mathrm{R}^{\prime}(\delta, 0, \mathrm{~m})=\max _{\Theta_{1}, \Theta_{2}\left(\Theta_{1}, \Theta_{2}, \mathrm{~m}\right) .}
\end{gathered}
$$

Hence the Theorem. 


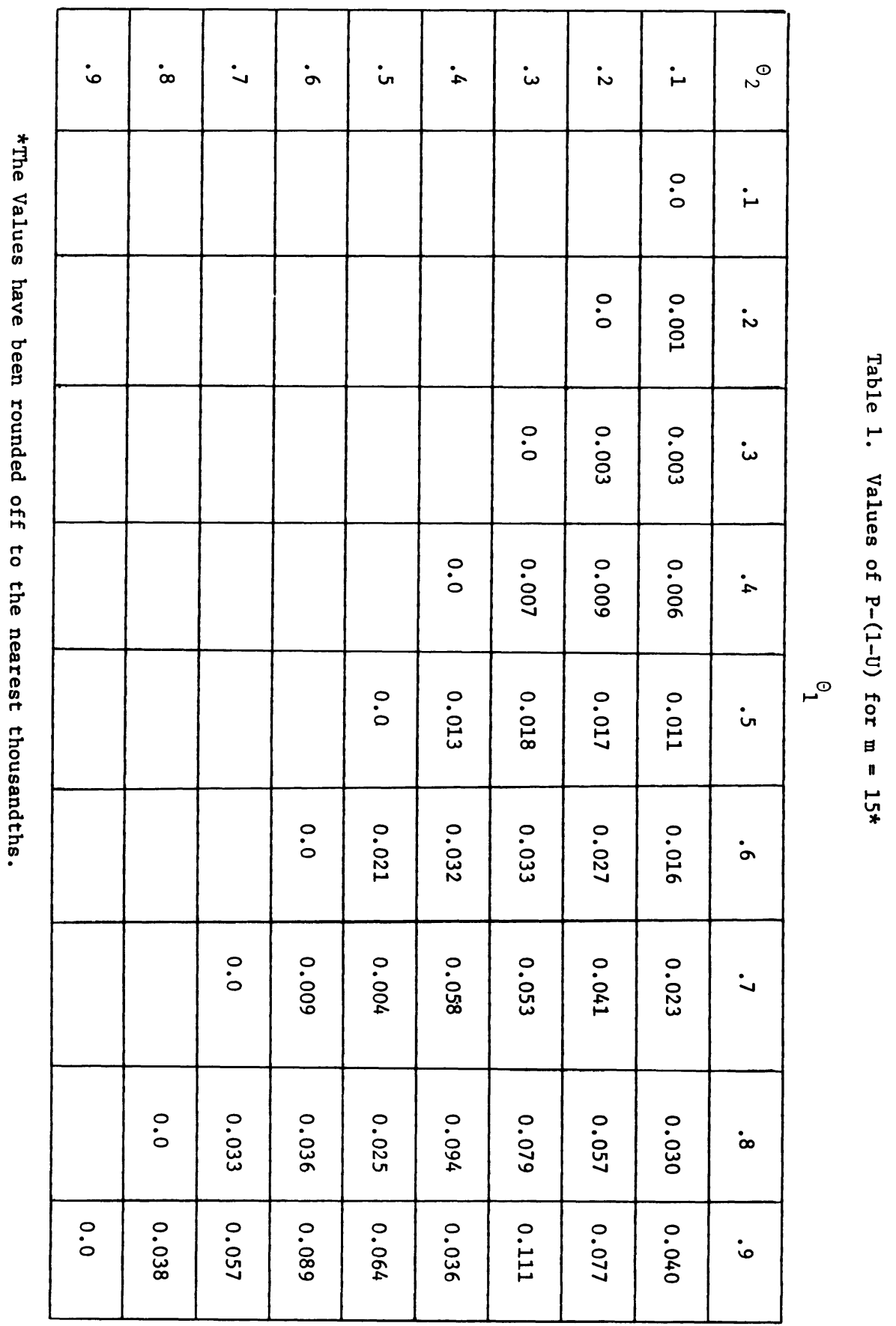




\section{COMPUTATIONAL RESULTS}

Table 1 gives the values of $P-(1-U)$ for $m=15$. In finding $(1-U)$ we have taken $n$ to be the integer closest to $E(S / 2)$. We observe that, for $\theta_{1}>\theta_{2}$, a11 the entries are positive. Since, for $\Theta_{1}>\Theta_{2},(P \star-P)$ is positive, it follows that P* - $(1-U)$ is positive and we can thus conclude that for all $\Theta_{1}>\Theta_{2}, R^{-}\left(\Theta_{1} \Theta_{2}, m\right) \geq$ $\mathrm{R}\left(\Theta_{1}, \Theta_{2}, \mathrm{~m}\right)$

The results for other values of $\mathrm{m}$ are almost identical and hence are not reported here. The effect of $\mathrm{m}$ is negligible since it appears in the expressions for $P$ and $\mathrm{E}(\mathrm{S})$ (See (2.1) and (2.3)) only as the power of $\left(\Theta_{1} \Theta_{2}\right)$.

Thus, based on this numerical evidence, a much stronger statement than Theorem 3.1 can be made, namely that, our procedure dominates the CPS - procedure over the entire $\left(\Theta_{1}, \Theta_{2}\right)$ - space.

\section{ACKNOWLEDGEMENTS}

The author thanks Mr. Michael Spevak for computational assistance and Professor Ajit Tamhane of Northwestern University for some useful discussions during the writing of this paper.

\section{REFERENCES}

1. CANNER, Paul L. Selecting One of Two Treatments when the Responses are Dichotomous. Journa1 of the American Statistical Association, 65 (1970), 293-306.

2. PRADHAN, MEENA and SATHE, Y.S. Analytical Remarks on Canner's Minimax Method for Finding the Better of Two Binomial Populations. Journal of the American Statistical Association, 71 (1976), 293-241.

3. SOBEL, MILTON and WEISS, GEORGE H. Play-the-Winner Sampling for Selecting the Better of Two Binomial Populations. Biometrika, 57 (1970), 357-365. 


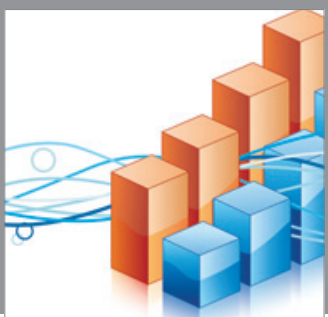

Advances in

Operations Research

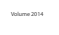

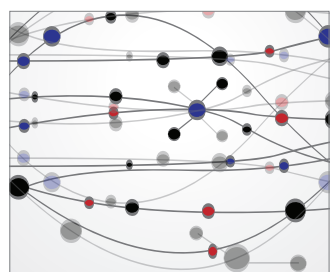

\section{The Scientific} World Journal
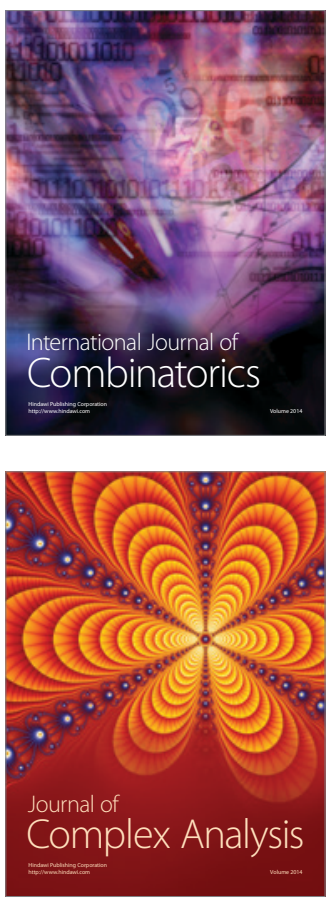

International Journal of

Mathematics and

Mathematical

Sciences
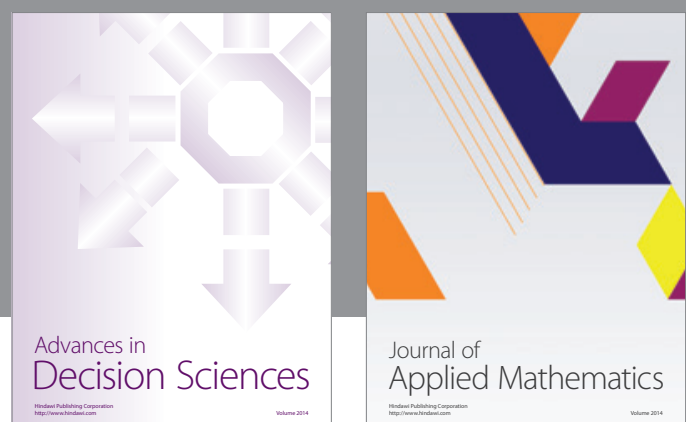

Journal of

Applied Mathematics
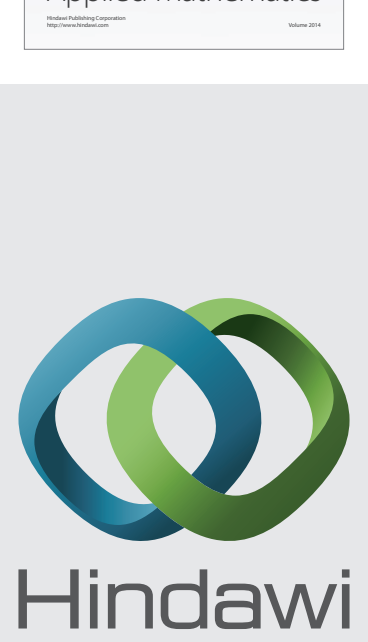

Submit your manuscripts at http://www.hindawi.com
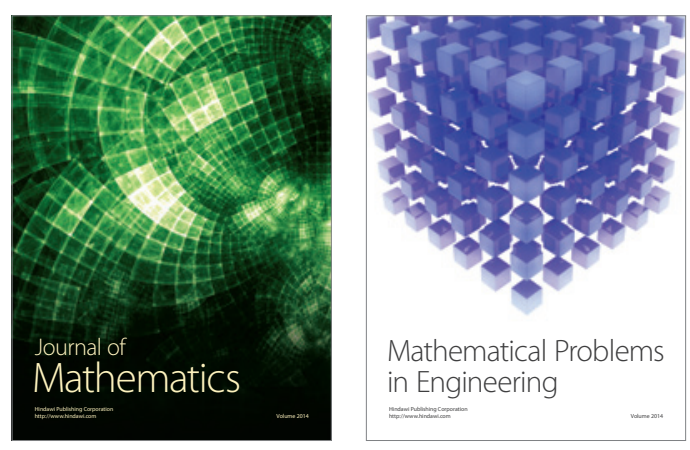

Mathematical Problems in Engineering
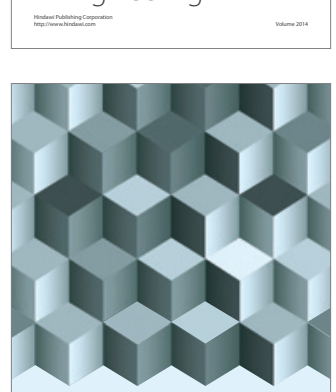

Journal of

Function Spaces
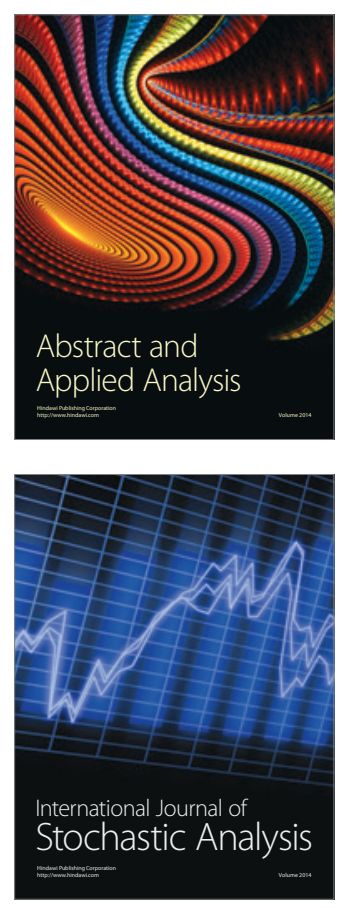

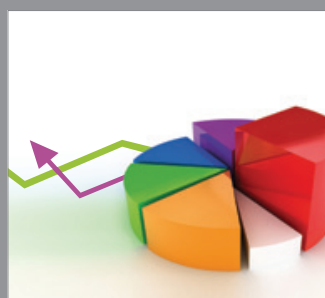

ournal of

Probability and Statistics

Promensencen
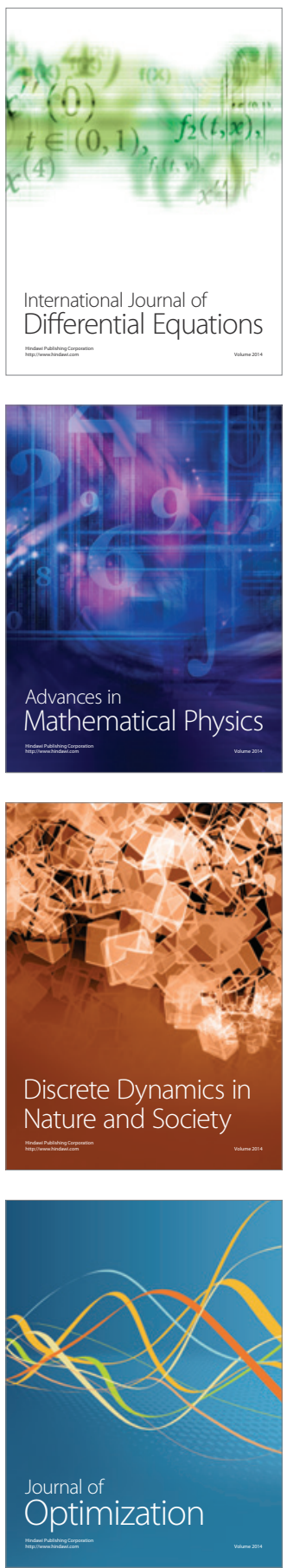